\begin{tabular}{|c|l|}
\hline Title & Hydrogen-assisted stabilization of Ni nanowires in solution \\
\hline Author(s) & Kiguchi, Manabu; Konishi, Tatsuy a; Murakoshi, Kei \\
\hline Citation & $\begin{array}{l}\text { APPLIED PHY SICS LETTERS, 87, 043104 } \\
\text { https://doi.org/40.1063/1.2001142 }\end{array}$ \\
\hline Issue Date & 2005-07 \\
\hline Doc URL & http://hdl.handle.net/2115/5913 \\
\hline Rights & Copyright $\odot 2005$ A merican Institute of Physics \\
\hline Type & article \\
\hline File Information & APL87-4.pdf \\
\hline
\end{tabular}

Instructions for use 


\title{
Hydrogen-assisted stabilization of Ni nanowires in solution
}

\author{
Manabu Kiguchi, Tatsuya Konishi, and Kei Murakoshia) \\ Department of Chemistry, Graduate School of Science, Hokkaido University, Sapporo 060-0810, Japan
}

(Received 8 March 2005; accepted 7 June 2005; published online 19 July 2005)

\begin{abstract}
We have studied conductance characteristics of mechanically fabricated Ni nanoconstrictions under controlling electrochemical potential and $p \mathrm{H}$ of the electrolyte. The conductance histogram showed a clear feature peaked at $1-1.5 G_{0}\left(G_{0}=2 e^{2} / h\right)$ when the potential of the constriction was kept at more negative potential than $-900 \mathrm{mV}$ versus $\mathrm{Ag} / \mathrm{AgCl}$ at $p \mathrm{H}=3.7$. A comparable feature also appeared at a more positive potential when a lower $p \mathrm{H}$ solution was used. We have revealed that $\mathrm{Ni}$ monoatomic contact or monoatomic wire can be stabilized in solution at room temperature under the hydrogen evolution. (C) 2005 American Institute of Physics. [DOI: 10.1063/1.2001142]
\end{abstract}

Construction of metal nanowires is the central issue of the current technology, because the techniques may be applied to fabricate ultimate electrical circuits in an atomic scale. Electrical conductance through a metal nanowire in an atomic scale is expressed by $G=2 e^{2} / h \sum T_{i}$; where $T_{i}$ is the transmission probability of the $i$ th conductance channel, $e$ is the electron charge, and $h$ is Plank's constant. Conductance quantization depends not only on the atomic structure of the nanoconstriction but also on inherent properties of the metal. ${ }^{1}$ Conductance at monoatomic point contact of transition metals with partially occupied $d$ orbitals is expected to be $1.5-3 G_{0}$ based on the theoretical calculation. ${ }^{1}$ Recently, quantized conductance of $\mathrm{Ni}$ nanoconstrictions has attracted much attention because of the expectation that a ferromagnetic nanowire may shows the conductance quantization in units of $0.5 G_{0}$, since there is no spin degeneracy. The upand down-spin electrons contribute independently to the electric transport. As well as $\mathrm{Ni}$, other ferromagnetic metals, $\mathrm{Fe}$ and $\mathrm{Co}$, have been extensively studied to confirm spindependent conductance quantization. However, previously documented data of experiments were less consistent with each other. Various conductance values, such as $1.6 G_{0}{ }^{2}{ }^{2}$ $G_{0}{ }^{3}$, and $0.5 G_{0}$, have been reported. ${ }^{4}$ In addition, a histogram of the conductance becomes featureless at room temperature. ${ }^{5}$ The poor agreement between former reported results may originate from the chemical instability of the nanoconstrictions of ferromagnetic metals. A novel experimental approach, under controlled chemical reactivity of ferromagnetic metals, is required to study the characteristics of the conductance quantization of ferromagnetic metals.

An electrochemical method has been recognized to also be another powerful approach to study the conductance quantization. ${ }^{6,7}$ Electrochemical potential determines the potential energy of electrons of the nanoconstriction, resulting in the control of the bonding strength between the metal atoms, and the interaction of the metals with molecules in a surrounding medium. The characteristics of the system lead to the successful fabrication of very stable metal nanostructures showing the conductance quantization which cannot be observed in an ultrahigh vacuum (UHV). For example, we reported that clear conductance quantization for Pd nanoconstrictions can be observed by the careful control of Pd depo-

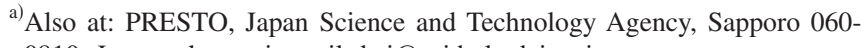
0810, Japan; electronic mail: kei@sci.hokudai.ac.jp sition and dissolution. ${ }^{7}$ It is noteworthy that conductance quantization was not observed in an UHV at room temperature. ${ }^{8}$ In this study, we have mechanically fabricated $\mathrm{Ni}$ nanoconstrictions in solution, and studied their conductance of the nanoconstrictions under controlled electrochemical potential and $p \mathrm{H}$ of the electrolyte. We discussed the effect of hydrogen on the stabilization of the Ni nanostructures, and the possibility of preparation of the $\mathrm{Ni}$ mono atomic wire in solution at room temperature.

The experiments were performed in an electrochemical cell mounted in a chamber that was filled with high-purity $\mathrm{N}_{2}$ gas $(>99.999 \%)$ to avoid the effect of oxygen in air. The tip was made of a $\mathrm{Au}$ wire (diameter $\sim 0.25 \mathrm{~mm},>99.9 \%$ ) coated with wax to reduce Faradic current between the tip and a counter electrode. The substrate was $\mathrm{Au}(111)$ prepared by flame annealing and quenching method. The electrochemical potential $\left(\Phi_{0}\right)$ of the Au substrate and tip was controlled using potentiostat (Pico-Stat, Molecular Imaging Co.), referring to a $\mathrm{Ag} / \mathrm{AgCl}$ reference electrode. The potential difference between the tip and substrate (bias voltage) was kept at $20 \mathrm{mV}$ for the conductance measurements. A Pt wire was used as a counterelectrode. The electrolyte was 10 $\mathrm{mM} \mathrm{NiSO}, 10 \mathrm{mM} \mathrm{H} \mathrm{BO}_{3}$, and $\mathrm{H}_{2} \mathrm{SO}_{4}$. The solution $p \mathrm{H}$ was adjusted by changing the concentration of $\mathrm{H}_{2} \mathrm{SO}_{4}$.

Nanoconstrictions of $\mathrm{Ni}$ were prepared in the following manner. First, Ni was electrochemically deposited both on a $\mathrm{Au}$ tip and a substrate by the polarization to negative potential than $\Phi_{0}=-650 \mathrm{mV}$ where $\mathrm{Ni}$ bulk deposition proceeded. ${ }^{9}$ After the confirmation of the bulk deposition of $\mathrm{Ni}$ on the tip and a substrate, the tip was pressed into the substrate and then pulling out of contact at a typical rate of $50 \mathrm{~nm} / \mathrm{s}$ in the electrochemical cell. During the contact breaking, a Ni nanoconstriction was formed between the tip and the substrate as schematically shown in Fig. 1.

Figure 2(a) shows the conductance histogram observed at $\Phi_{0}=-800 \mathrm{mV}$ in the electrolyte solution with a $p \mathrm{H}=3.7$. There was no clear feature in the conductance histogram, suggesting that any certain atomic configurations were not preferentially formed between Ni-deposited tip and a substrate. When the electrochemical potential of the nanoconstriction was kept more negative $\left(\Phi_{0}=-900 \mathrm{mV}\right)$, a clear feature appeared near 1-1.5 $G_{0}$ [see Fig. 2(b)]. Further polarization to the negative potential of a tip and a substrate did not change of the conductance histograms. The clear feature in the conductance histogram showing $1-1.5 G_{0}$ also ap- 


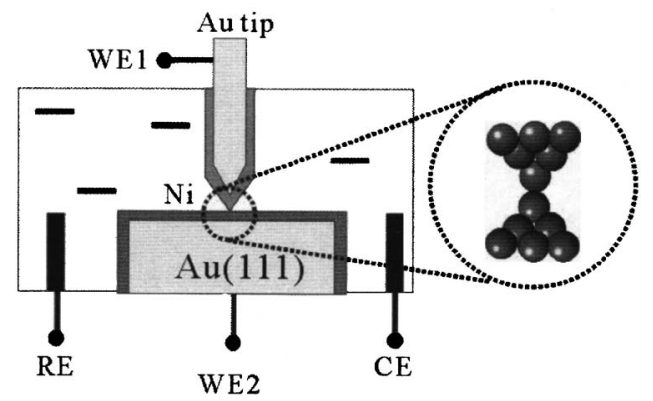

FIG. 1. Schematic presentation of a Ni nanoconstriction prepared under electrochemical-potential control. $\mathrm{Ni}$ is deposited on a Au single crystal substrate and a Au tip. RE, WE, and CE indicate reference electrode, working electrode, and counter electrode, respectively.

peared when the solution $p \mathrm{H}$ became lower than 2.7. Figure 2(c) shows the conductance histogram at $\Phi_{0}=-800 \mathrm{mV}$ at a $p \mathrm{H}=2.3$. A comparable feature to that of at $\Phi_{0}=-800 \mathrm{mV}$ and a $p \mathrm{H}=3.7$ was clearly observed in the histogram. A further decrease in $p \mathrm{H}$ did not change the conductance histogram. The potential, where a clear feature at $1-1.5 G_{0}$ was observed, corresponds to the hydrogen evolution region at the Ni electrode. ${ }^{10}$ Thus, the present result shows that the chemical reaction of hydrogen evolution significantly affects the conductance of $\mathrm{Ni}$ nanoconstriction in solution.

At the hydrogen evolution potential, the surface of $\mathrm{Ni}$ electrode is covered by the dissociated atomic hydrogen. ${ }^{10}$ The coverage of adsorbed hydrogen was evaluated by in situ infrared-reflection spectroscopy and the qualitative analysis of current-potential curves for hydrogen evolution. ${ }^{10,11}$ The coverage of adsorbed hydrogen gradually increases as the electrochemical potential becomes negative. Based on the results at the Ni polycrystalline electrode, the coverage of adsorbed hydrogen was estimated to be 0.20 and 0.40 at $\Phi_{0}=-800$ and $-900 \mathrm{mV}$ at a $p \mathrm{H}=3.7$, respectively. In more acidic solution with a $p \mathrm{H}=2.3$, comparable coverage can be achieved at a more positive potential by $100 \mathrm{mV}$. A comparable feature in the conductance histograms at a $p \mathrm{H}=3.7$ at $-900 \mathrm{mV}$ and that at a $p \mathrm{H}=2.3$ at $-800 \mathrm{mV}$ proves that the dissociated atomic hydrogen play an important role to define the geometrical and/or the electronic structure of the ferromagnetic metal nanoconstriction in solution.

It is noteworthy that the observed conductance histogram [Fig. 2(b)] was similar to that observed in UHV at an ultralow temperature. In an UHV, the clear $1-1.5 G_{0}$ feature appears in the conductance histogram at an ultralow temperature, ${ }^{12}$ while the histogram becomes entirely featureless at room temperature..$^{5}$ Thermal fluctuation of atoms at
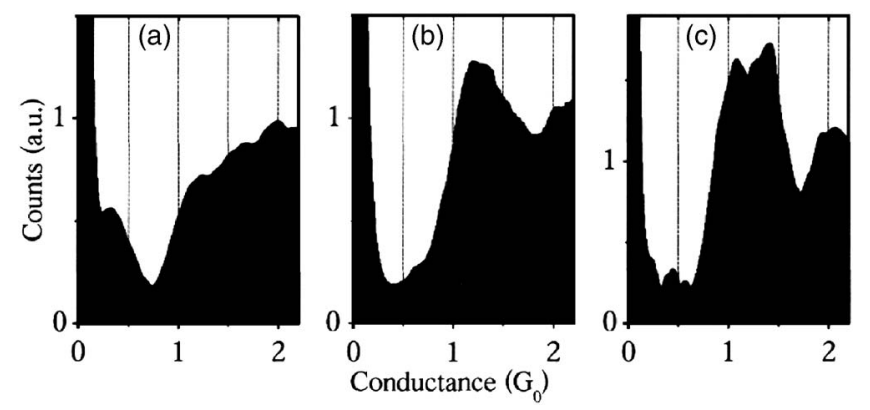

FIG. 2. Conductance histograms of Ni nanoconstrictions kept at (a) and (c) $\Phi_{0}=-800 \mathrm{mV}$, (b) $\Phi_{0}=-900 \mathrm{mV}$. Solution $p \mathrm{H}$ of the electrolytes was (a)

and (b) 3.7 and (c) 2.3.
Downloaded 08 Mar 2006 to 133.87.26.100. Redistribution subject to AlP license or copyright, see http://apl.aip.org/apl/copyright.jsp room temperature may prevent the long-term stability of the nanocontact, resulting in the broad feature in the histogram. At a low temperature in an UHV, thermal fluctuation is small. Thus, the nanostructure can have thermally stable structures showing specific values of the conductance. Although the fluctuation of atoms in solution at room temperature should be much larger than that in air and an UHV, the specific feature of the $\mathrm{Ni}$ nanoconstriction was found to be kept during the hydrogen evolution reaction even at room temperature. The results prove that the control of the electrochemical potential and solution $\mathrm{pH}$ leads to successful preparation of the Ni nanoconstrictions showing clear conductance quantization at room temperature.

At the present stage, it is not clear how the adsorbed atomic hydrogen stabilizes the certain atomic arrangement of the Ni nanoconstriction. The result proves that the effects of hydrogen on the conductance values in the present system are different from those in an UHV. In the previously documented result in an UHV, introducing hydrogen suppressed the characteristic conductance of $\mathrm{Ni}$ at $1-1.5 G_{0}$ by the replacement due to the evolution of a new value appearing near $1 G_{0}$ in the histogram. ${ }^{12}$ The origin of the value is a single hydrogen molecule bridging between two electrodes. ${ }^{13}$ In the case of $\mathrm{Pd}$, introducing hydrogen also suppresses the characteristic conductance of $1.8 G_{0}$, which gives new values appearing near 0.5 and $1 G_{0}$ in the histogram. ${ }^{14}$ These values of 0.5 and $1 G_{0}$ are attributable to a bridging single hydrogen molecule, and a $\mathrm{Pd}_{2} \mathrm{H}_{2}$ complex, respectively. On the other hand, in the present system in solution, the conductance histogram under the hydrogen evolution reaction was similar to that of a bare Ni metal in an UHV. The observation suggests that the hydrogen molecules did not bridge between the electrodes, despite the existence of the adsorbed hydrogen at the Ni surface.

Electrochemical potential control of the Ni nanoconstriction in solution leads to the control of the electronic density of the $\mathrm{Ni}$ atoms at the constriction. Characteristics of the electronic structure of the Ni nanoconstriction under the electrochemical potential control should be maintained the same as a bare $\mathrm{Ni}$ constriction in an UHV, even in the situation of the hydrogen adsorption on the surface. In addition to the effect of potential control of the system, the adsorbed hydrogen is known to alter the surface stress, because of modulation of the bonding strength between $\mathrm{Ni}$ atoms at the surface. ${ }^{15}$ In the present system, adsorbed hydrogen-as well as evolved hydrogen molecules in the vicinity of the $\mathrm{Ni}$ nanoconstriction - may help to stabilize the specific structure of the constriction mechanically via relatively weak physical and/or chemical interactions. These specific effects in the electrochemical system may lead to a comparable feature of the conductance histogram observed at the nanoconstriction of a bare $\mathrm{Ni}$ in an UHV at an ultralow temperature, even under the hydrogen evolution in solution at room temperature.

The conductance trace contains structural information on the $\mathrm{Ni}$ nanoconstriction in solution. Figure 3(a) shows the typical conductance trace at $\Phi_{0}=-800 \mathrm{mV}$ at a $p \mathrm{H}=3.7$. As a general tendency, the conductance change occurred continuously, rather than in a stepwise fashion. Although conductance occasionally showed small steps and plateaulike structures, their appearance and conductance values were nonreproducible, resulting in the featureless conductance histograms [see Fig. 2(a)]. Figures 3(b) and 3(c) show the typiAlP license or copyright, see http://apl.aip.org/apl/copyright.jsp 

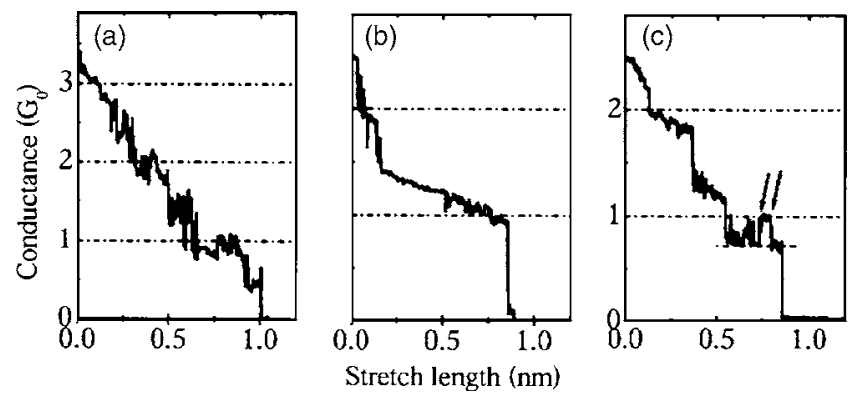

FIG. 3. Typical conductance traces of $\mathrm{Ni}$ nanoconstrictions observed at $\Phi_{0}=-800 \mathrm{mV}$ at a $p \mathrm{H}=3.7$ (a), and $p \mathrm{H}=2.3$ (b) and (c). Arrows in (c) indicate a reversible transition between $1 G_{0}$ and $0.6-0.7 G_{0}$ plateaus.

cal conductance trace at $\Phi_{0}=-800 \mathrm{mV}$ at a $p \mathrm{H}=2.3$. Although conductance change still occurred in a continuous fashion as at $\Phi_{0}=-800 \mathrm{mV}$ at a $p \mathrm{H}=3.7$, the conductance of $1-1.5 G_{0}$ was preferentially taken. It is the reason of an appearance of broad 1-1.5 $G_{0}$ feature in the histogram shown in Fig. 2(c).

At $\Phi_{0}=-800 \mathrm{mV}$ at a $p \mathrm{H}=2.3$, the plateau was stretched as long as $0.8 \mathrm{~nm}$ [see Fig. 3(b)]. Reversible transitions between $1 G_{0}$ and $0.6-0.7 G_{0}$ were occasionally observed in the long plateau [see Fig. 3(c)]. The conductance of a monoatomic contact is evaluated to be around $1.5-2.5 G_{0}$ for transition metals. ${ }^{1}$ Relatively long plateau and the conductance fluctuation at the plateau are the characteristics observed at the Ni nanoconstriction in solution. At the present stage, it is not clear whether the long plateau originated from the formation of the Ni monoatomic wire or it originated from deformation in a stem part of the nanoconstriction. This long plateau, however, implies the possibility of the formation of a monoatomic Ni wire in solution. The formation of a monoatomic wire may be supported by the reversible transition between $1 G_{0}$ and 0.6-0.7 $G_{0}$ [Fig. 3(c)]. A similar reversible transition of conductance between $1 G_{0}$ and 0.6-0.7 $G_{0}$ were reported for $\mathrm{Au}$ mononanoatomic wires in the presence of physically adsorbed hydrogen in an UHV at a low temperature $(10-30 \mathrm{~K}) .{ }^{16}$ The reversible transitions are explained by the dynamical structure transition between a dimerized $\mathrm{Au}$ wire and an equal-spacing wire. This dimerization is a characteristic of a monoatomic wire. ${ }^{17}$ Further detailed analysis of conductance traces and additional experiments are undergoing to clarify the origin of these conductance characteristics of the Ni nanoconstrictions in solution.
In conclusion, the dependence of the electrochemical potential and the solution $p \mathrm{H}$ on the conductance value was studied for the electrochemical deposited $\mathrm{Ni}$ nanoconstrictions. Under the hydrogen evolution reaction, a clear 1-1.5 $G_{0}$ feature appeared in the conductance histogram, suggesting that certain monoatomic arrangements were stabilized in solution. The analysis of the conductance trace showed the possibility of the formation of a one-dimensional Ni monoatomic wire in solution at room temperature. This hydrogenassisted stabilization of metal nanostructure can be applied as an effective method to control the structure of the nanoconstriction in an atomic scale, and fabricate various interesting structures for the study of one-dimensional systems.

This work was partially supported by the Grant-in-Aid for Scientific Research from the Ministry of Education, Culture, Sports, Science, and Technology, Japan.

${ }^{1}$ N. Agrait, A. L. Yeyati, and J. M. van Ruitenbeek, Phys. Rep. 377, 81 (2003).

${ }^{2}$ C. Sirvent, J. G. Rodrigo, S. Vieira, L. Jurczyszyn, N. Mingo, and F. Flores, Phys. Rev. B 53, 16086 (1996).

${ }^{3}$ Y. Ooka, T. Ono, and H. Miyajima, J. Magn. Magn. Mater. 226, 1848 (2001).

${ }^{4}$ F. Elhoussine, S. Matefi-Tempfli, A Encinas, and L. Piraux, Appl. Phys. Lett. 81, 1681 (2002).

${ }^{5}$ J. L. Costa-Krämer, Phys. Rev. B 55, R4875 (1997).

${ }^{6}$ C. Shu, C. Z. Li, H. X. He, A. Bogozi, J. S. Bunch, and N. J. Tao, Phys. Rev. Lett. 84, 5196 (2000).

${ }^{7}$ J. Li, T. Kanzaki, K. Murakoshi, and Y. Nakato, Appl. Phys. Lett. 81, 123 (2002).

${ }^{8}$ A. Enomoto, S. Kurokawa, and A. Sakai, Phys. Rev. B 65, 125410 (2002).

${ }^{9}$ M. Zhou, N. Myung, X. Chen, and K. Rajeshwar, J. Electroanal. Chem. 398, 5 (1995).

${ }^{10}$ G. Kreysa, B. Hakansson, and P. Ekdunge, Electrochim. Acta 33, 1351 (1988).

${ }^{11}$ J. O. Bockris, J. L. Carbajal, B. R. Scharifker, and K. Chandrasekaran, J. Electrochem. Soc. 134, 1957 (1987).

${ }^{12}$ C. Untiedt, D. M. T. Dekker, D. Djukic, and J. M. van Ruitenbeek, Phys. Rev. B 69, 081401 (2004).

${ }^{13}$ R. H. M. Smit, Y. Noat, C. Untiedt, N. D. Lang, M. C. van Hemert, and J. M. van Ruitenbeek, Nature (London) 419, 909 (2002).

${ }^{14}$ S. Csonka, A. Halbritter, and G. Miha'ly, O. I. Shklyarevskii, S. Speller, and H. van Kempen, Phys. Rev. Lett. 93, 016802 (2004).

${ }^{15}$ S. Armyanov and G. S. Sotirova-Chakarova, Met. Finish. 91, 59 (2003).

${ }^{16}$ Sz. Csonka, A. Halbritter, G. Mihaly, E. Jurdik, O. I. Shklyarevskii, S. Speller, and H. van Kempen, Phys. Rev. Lett. 90, 116802 (2003).

${ }^{17}$ M. Okamoto and K. Takayanagi, Phys. Rev. B 60, 7808 (1999). 